\title{
Fractal lacunarity of trabecular bone in vertebral MRI to predict osteoporotic fracture risk in over-fifties women. The LOTO study
}

Annamaria Zaia ${ }^{1 *} \mathbb{D}$, Roberto Rossi ${ }^{2}$, Roberta Galeazzi ${ }^{3}$, Manuela Sallei ${ }^{2}$, Pierluigi Maponi ${ }^{4}$ and Pietro Scendoni ${ }^{5}$

\begin{abstract}
Background: Osteoporotic fractures are a major cause of morbidity in the elderly. Menopausal women represent the population with the highest risk of early osteoporosis onset, often accompanied by vertebral fractures (VF). Bone mineral density (BMD) is commonly assessed by dual-energy X-ray absorptiometry (DXA) for osteoporosis diagnosis; however, BMD alone does not represent a significant predictor of fracture risk. Bone microarchitecture, instead, arises as a determinant of bone fragility independent of BMD. High-resolution magnetic resonance imaging (MRI) is an effective noninvasive/nonionizing tool for in vivo characterisation of trabecular bone microarchitecture (TBA). We have previously set up an MRI method able to characterise TBA changes in aging and osteoporosis by one parameter, trabecular bone lacunarity parameter $\beta(T B L \beta)$. Fractal lacunarity was used for TBA texture analysis as it describes discontinuity of bone network and size of bone marrow spaces, changes of which increase the risk of bone fracture. This study aims to assess the potential of TBL $\beta$ method as a tool for osteoporotic fracture risk.
\end{abstract}

Methods: An observational, cross-sectional, and prospective study on over-50s women at risk for VF was designed. TBL $\beta$, our index of osteoporotic fracture risk, is the main outcome measure. It was calculated on lumbar vertebra axial images, acquired by 1.5 T MRI spin-echo technique, from 279 osteopenic/osteoporotic women with/without prior VF. Diagnostic power of TBL $\beta$ method, by Receiver Operating Characteristics (ROC) curve and other diagnostic accuracy measurements were compared with lumbar spine DXA-BMD.

Results: Baseline results show that TBL $\beta$ is able to discriminate patients with/without prevalent VF $(p=0.003)$. AUC (area under the curve from $\mathrm{ROC}$ ) is 0.63 for $\mathrm{TBL} \beta$, statistically higher $(p=0.012$ ) than BMD one (0.53). Contribution of TBL $\beta$ to prevalent VF is statistically higher $(p<0.001)$ than BMD (sensitivity: $66 \%$ vs. $52 \%$ respectively; OR: 3.20, $p<$ 0.0001 for TBL $\beta$ vs. 1.31, $p=0.297$ for BMD). Preliminary 1-year prospective results suggest that TBA contribution to incident VF is even higher (sensitivity: $73 \%$ for TBL $\beta$ vs. $55 \%$ for BMD; RR: 3.00, $p=0.002$ for TBL $\beta$ vs. 1.31, $p=0.380$ for BMD).

(Continued on next page)

\footnotetext{
* Correspondence: a.zaia@inrca.it

${ }^{1}$ Centre of Innovative Models for Ageing Care and Technology, Scientific

Direction, IRCCS INRCA, Via S. Margherita 5, I-60121 Ancona, Italy

Full list of author information is available at the end of the article
}

(c) The Author(s). 2021 Open Access This article is licensed under a Creative Commons Attribution 4.0 International License, which permits use, sharing, adaptation, distribution and reproduction in any medium or format, as long as you give appropriate credit to the original author(s) and the source, provide a link to the Creative Commons licence, and indicate if changes were made. The images or other third party material in this article are included in the article's Creative Commons licence, unless indicated otherwise in a credit line to the material. If material is not included in the article's Creative Commons licence and your intended use is not permitted by statutory regulation or exceeds the permitted use, you will need to obtain permission directly from the copyright holder. To view a copy of this licence, visit http://creativecommons.org/licenses/by/4.0/ The Creative Commons Public Domain Dedication waiver (http://creativecommons.org/publicdomain/zero/1.0/) applies to the data made available in this article, unless otherwise stated in a credit line to the data. 
(Continued from previous page)

Conclusion: Results from this study further highlight the usefulness of TBL $\beta$ as a biomarker of TBA degeneration and an index of osteoporotic fracture risk.

Keywords: Osteoporosis, Fracture risk, Vertebral fracture, Trabecular bone microarchitecture, Magnetic resonance imaging, Fractal lacunarity, Bone mineral density

\section{Background}

Osteoporosis, as defined by World Health Organization (WHO), is a systemic skeleton disease characterised by low bone mass and microarchitectural deterioration of bone tissue with consequent increase of bone fragility and susceptibility to fracture [1]. This pathology can be induced by a variety of causes and has been classified in either primary or secondary osteoporosis. Primary osteoporosis is strictly linked to age-related deterioration of bone tissue while secondary osteoporosis can be the consequence of various conditions and diseases or can be induced by medications that adversely affect bone health. Primary or primitive osteoporosis has been further classified in type I (postmenopausal) and type II (senile) osteoporosis. Type I osteoporosis typically affects 50-65 years old women due to accelerated trabecular bone resorption linked to oestrogen deficiency. Type II (senile) osteoporosis is peculiar to over-65 s subjects when cortical bone deterioration is also involved. The fracture pattern in postmenopausal osteoporosis mainly involves the spine while senile osteoporosis is characterised by fractures mainly affecting hip and femur [2].

Osteoporotic fractures are a major cause of morbidity in the elderly and menopausal women represent the population with the highest risk of early osteoporosis onset. The increased lifespan in the industrialized world accounts for increasing incidence of osteoporosis and bone fractures with a perspective of additional years (at least 20) of disability osteoporotic women have to face in their later life $[3,4]$. The dimension and complexity of bone fragility problem are huge: in Europe, osteoporosis causes every year almost 1 million hip fractures, 500,000 femur fractures, and 1,400,000 vertebral fractures (VF) over those to other sites (wrist, humerus, ribs). Hip fractures $(75 \%)$ and VF (85\%) mainly affect women. The remaining lifetime probability in women at the menopause of a fracture at any site exceeds that of breast cancer (approximately 12\%). The likelihood of a fracture at any of these items is $40 \%$ or more in developed countries, a picture close to the probability of coronary heart disease [4-6].

Due to the silent progression of bone structure degeneration, osteoporosis diagnosis often follows a painful fracture event. Currently, only a small percentage of individuals knows to be osteoporotic while the condition of most pathologic people remains undiagnosed until a fracture occurs [7]. VF may cause acute pain and loss of function, but may also occur without serious symptoms; however, they often recur, and the consequent disability increases with the number of fractures. It is worth noting that the first fracture event increases the risk and accelerates the onset of new ones [8].

The estimate of areal bone mineral density (BMD) by means of dual-energy x-ray absorptiometry (DXA) represents the most common approach to diagnose osteoporosis and predict fracture risk. However, BMD alone is not a good predictor for fracture risk $[9,10]$. Over the past decades, it has been recognized that factors of bone quality also contribute to fracture risk. In particular, microarchitecture emerges as a determinant of bone strength independent of BMD $[11,12]$ and its investigation would give insight into the mechanisms of bone fragility as well as the action of drugs used to prevent osteoporotic fractures [11].

The rapid spreading of medical imaging techniques in clinical practice, together with the impressive development of information technologies, has solicited proliferation of new methods for in vivo assessment of trabecular bone microarchitecture (TBA) changes with ageing and osteoporosis [13, 14]. Developments in high-resolution magnetic resonance imaging (MRI) techniques have expanded perspectives for in vivo characterisation of TBA by noninvasive/nonionizing methods [15]. Nevertheless, texture analysis, mainly based on classic histomorphometric methods, is not frequently used in clinical practice because of the large number of calculated parameters that makes difficult their interpretation.

An original and innovative method of MR image analysis, developed in our Institute, has been previously proposed to provide a unique parameter sensitive to TBA changes in ageing and osteoporosis [16, 17]. It has been set up by considering the complexity of human beings and fractal properties of several anatomic and physiologic structures among which is bone tissue [14, 18-23]. Characterising TBA by fractal lacunarity seems to be a suitable approach. Fractal lacunarity, in fact, by measuring space-filling capacity of a complex object, has the potential to describe both bone network discontinuity and sizes of trabecular spaces (bone marrow) [17, 24], changes of which represent an index of increased fracture risk. The mathematical solution proposed is one parameter calculated from the simple hyperbola formula 
that well fits the curvilinear plot obtained from lacunarity analysis of trabecular network [14, 16, 17, 25]. In previous studies, we observed that parameter $\beta$, representative of lacunarity, correlates with both age and physio-pathologic status $[14,16,17,25]$. Therefore, parameter $\beta$ (namely TBL $\beta$ : trabecular bone lacunarity parameter $\beta$ ) is the natural candidate to become a standard for TBA characterisation and a potential index of bone fragility fracture risk.

Here we present an observational, cross-sectional, and prospective study on over-50s women at risk for osteoporotic fractures designed for diagnostic power assessment of this potential new diagnostic tool. In particular, baseline results from LOTO (Lacunarity Of Trabecular bone in Osteoporosis) study are described and discussed. Preliminary 1-year prospective results are also presented.

\section{Methods}

\section{Study design and participants}

An observational, cross-sectional, and prospective study was designed to assess the diagnostic power of the new tool, potentially useful for early diagnosis of fracture risk in osteoporotic pathology. It is based on fractal lacunarity analysis of TBA in MRI lumbar vertebra images.

Osteopenic/osteoporotic over-50s women, at risk for bone fragility-spontaneous fractures, were recruited for baseline assessment as described below. Follow up at 1 , 2 and 5 years have been also planned.

The main objective of the present study is to verify the potential of TBL $\beta$ in discriminating patients with and without bone fragility VF by assessing diagnostic power of TBL $\beta$ method in baseline data. The main outcome measure is TBL $\beta$ of our bio-mathematical model as an index of osteoporotic fracture risk. It was calculated by means of a software prototype, developed by adopting a greyscale version of the method [14] outlined below, on L4 axial section images acquired by $1.5 \mathrm{~T}$ MRI spin-echo multislice technique. DXA-BMD at L1-L4 lumbar spine was used as the reference standard.

\section{Ethical standards}

The LOTO study was approved by the institutional Ethical Committee (FiORdiLOTO SC/11/281) and was performed in accordance with the Declaration of Helsinki (1964) and its later amendments.

Written informed consent was received from participants prior to inclusion in the study.

\section{Procedures}

After approval by the Ethics Committee of our Institute, over-50s women defined osteopenic/osteoporotic on the bases of DXA T-score at L1-L4 lumbar spine were enrolled among those who asked for our Institute BMD-
Service based on the following inclusion/exclusion criteria.

Patients were enrolled when eligible for all the following characteristics and conditions: consecutive patients who accepted to be clinically followed by our Bone Metabolism Unit; age of 50 years and older; densitometric diagnosis of osteopenia ( $\mathrm{T}$-score between -1 and -2.5 ) or osteoporosis (T-score equal to -2.5 or lower) at L1L4 lumbar spine; primitive (postmenopausal and senile) osteoporosis, with or without prior vertebra bone fragility fractures; have given written informed consent.

Patients were excluded when at least one of the following conditions or pathologies was present: osteoporosis secondary to drug-induced bone loss, chronic diseases, or genetic diseases; contraindications to MRI; severe impairment of cognitive/functional status.

After first visit and interview to record demographic and clinical data, patients underwent the regular diagnostic practice for osteoporosis and osteoporotic fractures (DXA, blood analysis, dorsal-lumbar spine $\mathrm{x}$-ray). Patients characterised as described above underwent lumbar spine MRI to acquire spin-echo axial multislice vertebral images for TBA characterisation.

\section{Bone mineral density assessment}

BMD measurement to classify patients in terms of osteopenia/osteoporosis was performed by means of a DXA fan bean system (low x-ray emission) (Lunar Prodigy Primo - GE Medical Systems). Densitometry reports were based on WHO criteria for DXA lumbar and femoral scans by expressing results as T-score and Z-score. In particular, based on T-score values at L1-L4 lumbar spine, patients' BMD was defined as: normal (T-score $\geq$ $-1 \mathrm{SD}$ ); low bone mass (osteopenia) (T-score between -1 and - 2.5 SD); osteoporosis (T-score $\leq-2.5 \mathrm{SD}$ ). Zscore, reflecting bone density compared with other people in the same age-group and of the same size and gender, was considered to exclude secondary osteoporosis. In fact, if this score is unusually high or low, it may indicate that factors other than age can affect BMD.

\section{X-ray of dorsal-lumbar spine}

A conventional $\mathrm{x}$-ray apparatus with remote control (Prestige-GE Medical Systems) was used to acquire images of dorsal-lumbar spine to be interpreted by morphometry techniques for the diagnosis of VF. Exposure technique of spine in lateral projection was applied according to Genant criteria [26] for morphometry analysis. A computed radiography system with morphometry software for visualization/analysis (Medstation-Exprivia) was used and the quantitative method was applied. It allows fracture diagnosis based on both reduction of $4 \mathrm{~mm}$ threshold value and $15 \%$ reduction of at least one height of vertebral body. Morphometry on 
conventional $\mathrm{x}$-ray images (MRX) has been preferred to that on densitometric images (MXA) as it represents the best approach for the diagnosis of osteoporotic fracture prevalence. MRX allows obtaining both semiquantitative and quantitative evaluation with higher precision than MXA [26].

\section{Magnetic resonance imaging of lumbar spine}

High resolution MRI, $1.5 \mathrm{~T}$ whole body system (Gyroscan Intera; Philips-Medical System, ACR-Nema 1.0), was used for dorsal-lumbar spine MRI scan using a phased array dS Spine coil. Spin-echo multislice technique was applied for the acquisition of TBA axial section images of vertebral bodies ( 9 to 12 slices with a thickness of $3 \mathrm{~mm}$ without space gap between slices). The pulse sequence was as follow: TE of $15 \mathrm{~ms}$, TR of $525 \mathrm{~ms}$; flip angle was of $90^{\circ}$, matrix of $512 \times 512$, and pixel size equal to $0.469 \mathrm{~mm}$ for a scan time below 15 min. To confirm the reproducibility of this image acquisition method assessed in previous studies, the pulse sequence was initially tested on two subjects undergone lumbar spine MRI scan for three times in two weeks. As previously reported $[14,16,17]$, the method was set up on real images to avoid failure that often occurs when phantom and/or simulation are used to develop new methods. Spin-echo technique was systematically applied to L1-L4 lumbar spine.

\section{Estimate of TBL $\beta$ in magnetic resonance images}

Computation of TBL $\beta$, potential index of fracture risk, was performed on MR spin-echo images of lumbar vertebras by adopting a method previously developed in our laboratory as described in $[16,17]$ and modified in $[14$, 25].

Briefly, to estimate lacunarity we chose the gliding box algorithm, GBA, based on the analysis of mass (M) distribution in binary images [27]. A simple extension of this algorithm was used to deal with greyscale images. In this extension, the moment formula for the discrete distribution of $\mathrm{M}$ was upgraded with the moment formula for $\mathrm{M}$ taking a continuous range of possible values, see $[14,25]$ for details. The efficiency of such an extension was improved by a different pre-processing step through a sigmoid function to weight grey level of each pixel.

The GBA method was implemented in software, by using MATLAB software package (the MatWorks, Inc.), to operate on both binary images, after a pre-processing step involving greyscale reversion (version 1) [16, 17] and on original greyscale images with and without reversed greyscale (versions 2 and 3) [14, 25]. In these versions, a sigmoid function is used for grey levels' rescaling. This procedure allows weighting grey level for each pixel, thus limiting the risk of information loss due to image binarization. Version 2 was considered in the present study, as it is more sensitive than version 1 and more robust than version 3 [14].

Figure 1 shows a schematic representation of the method. In particular, the following model:

$$
L(b)=\frac{\beta}{b^{\alpha}}+\gamma, b \in\left[b_{\min }, b_{\max }\right]
$$

was chosen to approximate the hyperbola-like curvilinear plot of lacunarity function $\mathrm{L}(\mathrm{b})$ where $\alpha, \beta, \gamma$ are suitable parameters $[16,17]$. Dealing with fractals, $\alpha$ is related to the fractal dimension of the set and $\beta$ (our TBL $\beta$ ) characterises the lacunarity of the set [14, 27]. Low TBL $\beta$ values correspond to high lacunarity, that is, high deterioration of TBA that predisposes to increased fracture risk.

The standardized version of our method was set up by using the middle axial section(s) of L4. Lacunarity analysis on the other sections of the same vertebra and on other vertebras (i.e. L2) was performed to further validate the standardized version or to suggest further improvements. Intra- and inter-operator variability was confirmed always lower than $2 \%$ by randomly repeating twice ROI selection and lacunarity estimate.

\section{Sample size and statistical analyses}

Taking into account the primary objectives of LOTO study, that is, diagnostic capacity and accuracy of TBL $\beta$ method, the sample size was estimated of 280 osteopenic/osteoporotic patients, 224 without VF and 56 with prior VF based on a $20 \%$ prevalence of VF in over-50s women, a non-relevant incidence in two-year follow up, and a $10 \%$ drop out. Sample size was calculated based on: 0.05 first type error; more than $80 \%$ study power; AUC (area under the curve) in ROC (receiver operating characteristics) curve analysis for diagnostic accuracy measurement; 0.80 high level of diagnostic accuracy; 0.10 moderate difference between TBL $\beta$ and DXA-BMD; $1 / 4$ patient ratio with/without VF; a moderate variability among observers.

Statistical analyses were performed by means of SPSS package v. 19 (SPSS Inc. Chicago IL) and statistical significance was accepted for $p \leq 0.05$. Descriptive statistics were used to synthesize demographic and clinicalpathologic characteristics of the whole patients' sample. Univariate analysis was performed for continuous variables. Student's $t$-test and Chi-squared test were used to compare differences between groups. Nonparametric alternative Mann-Withney $U$ test was used when normality assumption, checked by the Kolmogorov-Smirnov test, was not valid. Diagnostic accuracy of TBL $\beta$ method in predicting VF was evaluated by ROC curve analysis (AUC) that provides a combined measure of sensitivity (SN) and specificity (SP). Statistical significance of 

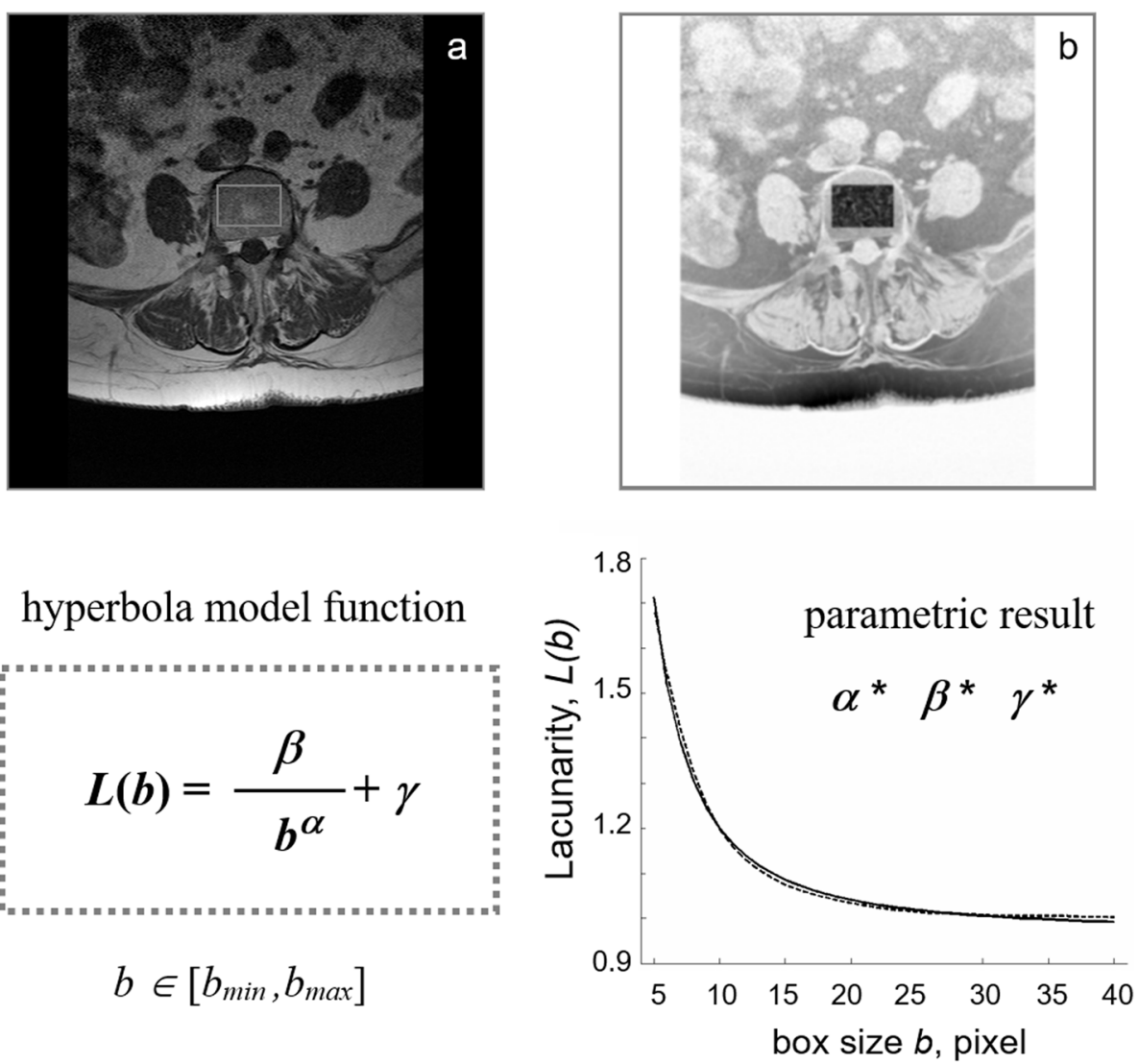

Fig. 1 Schematic representation of TBL $\beta$ method. a 1.5 T MRI spin-echo image $(512 \times 512$ pixel, pixel size equal to $0.469 \mathrm{~mm})$ of fifth out of nine axial section of the 4th lumbar vertebra; $\mathbf{b}$ Rectangular $\mathrm{ROI}$ within the inner perimeter of vertebral body in an intermediate step of image processing on reverse greyscale image. The plot (right bottom) represents the result of gliding box algorithm application (dotted line) as fitted by hyperbola model function (solid line) used to calculate the triplet of parameters $a^{*}, \beta^{*}, \gamma^{*}$ (left bottom). TBL $\beta$ : trabecular bone lacunarity parameter $\beta$; MRI: magnetic resonance imaging; ROI: region of interest

discriminating power of the test was defined by zeta test. The best cut-off values of TBL $\beta$ to predict VF, defined by Youden index in ROC curve and by median value from the whole sample, were used to calculate also other diagnostic accuracy measurements (odds ratio, OR; relative risk, $\mathrm{RR}$ and attributable risk, $\mathrm{AR}$; $\mathrm{SN}, \mathrm{SP}$, positive and negative predictive values (PPV and NPV), and effectiveness (accuracy, ACC).

\section{Results}

\section{Demographic and clinical characteristics}

A complete dataset of baseline recording was obtained for 279 out of 315 subjects eligible for the study (Fig. 2). Table 1 summarizes main demographic and clinical characteristics of the whole sample and of the two subgroups considered: VF+, with prevalent VF (n 88, 32\%) and VF-, without VF (n 191, 68\%).

The age range of the whole sample was $50-85$ years (mean age \pm SD equal to $60 \pm 7$, median 59). A similar age distribution was observed in VF- (mean age $59 \pm 7$ years, range $50-85$, median 58 ) while $\mathrm{VF}+$ showed a statistically higher mean age (62 \pm 7 years, range $51-80$, median $62, p=0.001$ ) when compared with VF-.

BMD T-score at L1-L4 lumbar spine was found equal or lower than $-2.5 \mathrm{SD}$ (osteoporosis) in $47.7 \%$ women. Prevalent VF were found in $31.5 \%$ subjects, $47.7 \%$ of which defined osteopenic at lumbar spine by DXA-BMD and $67 \%$ younger than 65 years. Figure 3 shows the prevalence of VF within both age and BMD subgroups.

The last, but not least, discrimination regarding the sample under study deals with osteoporosis treatment. We found that 123 out of 279 patients (44\%) were with at least one osteoporosis medication at baseline, 77\% of which with Vitamin D and/or Calcium supplements and $57 \%$ under bisphosphonates treatment (with or without Vitamin D/Calcium supplements), while other osteoporosis medications accounted for only $8 \%$. Among overall treated $(\mathrm{T}+)$ subjects, $57 \%$ were VF- (mean age $62 \pm 7$ years; range $51-85)$ and $43 \%$ were $\mathrm{VF}+(63 \pm 8$ years; range 51-80) while untreated (T-) subjects accounted for $78 \%$ VF- (mean age $58 \pm 6$ years; range $50-81$ ) and $22 \% \mathrm{VF}+$ (mean age $61 \pm 7$ years; range $51-74$ ). 


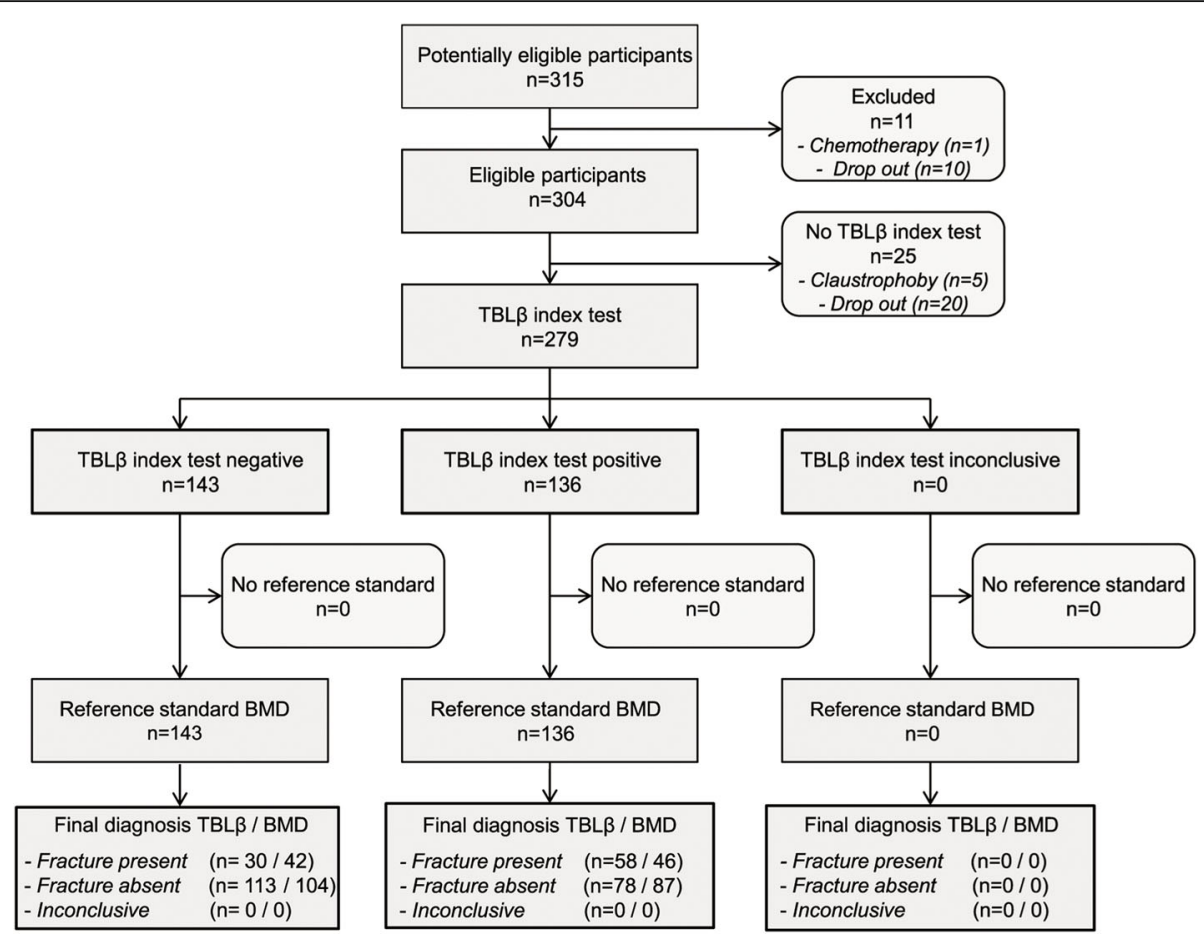

Fig. 2 Flow chart of LOTO study to assess diagnostic accuracy of TBL $\beta$ vs. BMD. TBL $\beta$ : trabecular bone lacunarity parameter $\beta$; BMD: bone mineral density

TBL $\beta$ as an index of osteoporotic fracture risk

TBL $\beta$ was calculated as the mean value of the two central L4 axial sections (i.e. 5th and 6th of 10). Figure 4 shows age-related distribution of TBL $\beta$ and lumbar spine BMD in VF+ and VF- subjects. TBL $\beta$ values were not normally distributed; therefore, results in Table 2 were expressed as median (interquartile range) and $p$ values were estimated by Mann-Whitney $U$ test. Results from the whole sample showed that TBL $\beta$ is able to discriminate between VF+ and VF- with values statistically

Table 1 Demographic and clinical characteristics of LOTO patients

\begin{tabular}{|c|c|c|c|}
\hline \multirow[t]{2}{*}{ Characteristic } & Overall & without VF & with VF \\
\hline & $N=279$ & $N=191$ & $N=88$ \\
\hline Age, years & $60 \pm 7$ & $59 \pm 7$ & $62 \pm 7$ \\
\hline$<65$ & $212(76.0)$ & $153(80.1)$ & $59(67.0)$ \\
\hline$\geq 65$ & $67(24.0)$ & $38(19.9)$ & $29(33.0)$ \\
\hline Body mass index, $\mathrm{kg} / \mathrm{m}^{2}$ & $23.09 \pm 3.00$ & $22.98 \pm 2.94$ & $23.32 \pm 3.14$ \\
\hline$\leq 25$ & $217(77.8)$ & $152(79.6)$ & $65(73.9)$ \\
\hline$>25$ & $62(22.2)$ & $39(20.4)$ & $23(26.1)$ \\
\hline Bone mineral density, L1-L4T-score & $-2.4 \pm 0.9$ & $-2.4 \pm 0.9$ & $-2.5 \pm 0.8$ \\
\hline$\leq-2.5$ & $133(47.7)$ & $87(45.5)$ & $46(52.3)$ \\
\hline$>-2.5$ & $146(52.3)$ & $104(54.5)$ & $42(47.7)$ \\
\hline \multicolumn{4}{|l|}{ Osteoporosis medication } \\
\hline any medication & $123(44.1)$ & $70(36.6)$ & $53(60.2)$ \\
\hline Vitamin D and/or Calcium supplements ${ }^{a}$ & $95(77.2)$ & 55 (78.6) & $40(75.5)$ \\
\hline Bisphosphonates $^{a}$ & $70(56.9)$ & $43(61.4)$ & $27(50.9)$ \\
\hline other ${ }^{a}$ & $10(8.1)$ & $3(4.3)$ & $7(1.3)$ \\
\hline
\end{tabular}

Data are represented as mean \pm SD or $n(\%)$. VF: prevalent vertebral fracture

a Percentage calculated within treated patients' group 

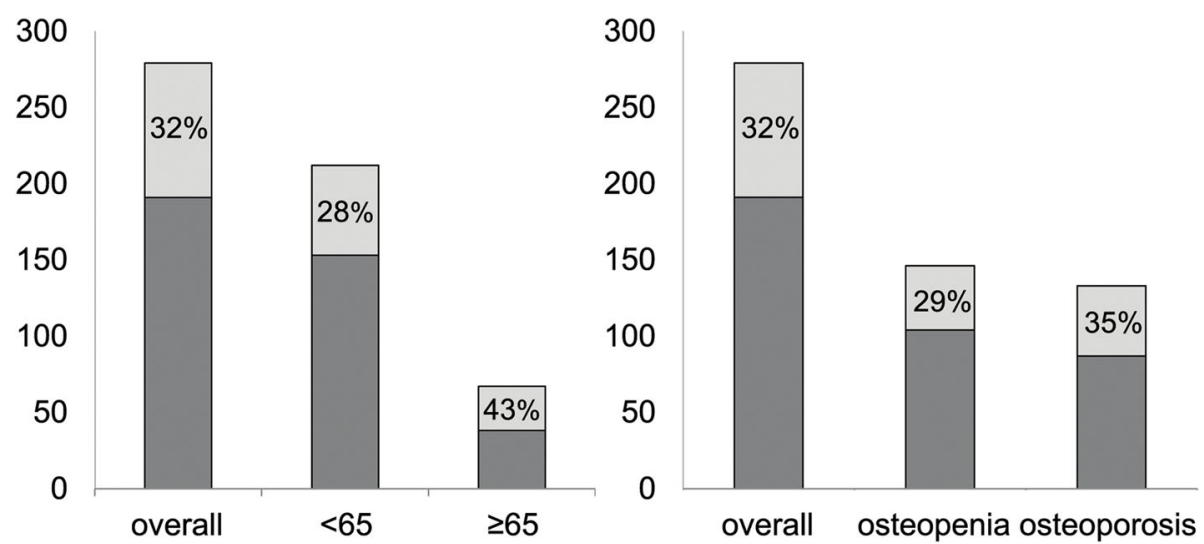

Fig. 3 Prevalence of bone fragility vertebral fractures (light grey) in osteopenic/osteoporotic over-50s women related to both age (left) and BMD (right). BMD: bone mineral density

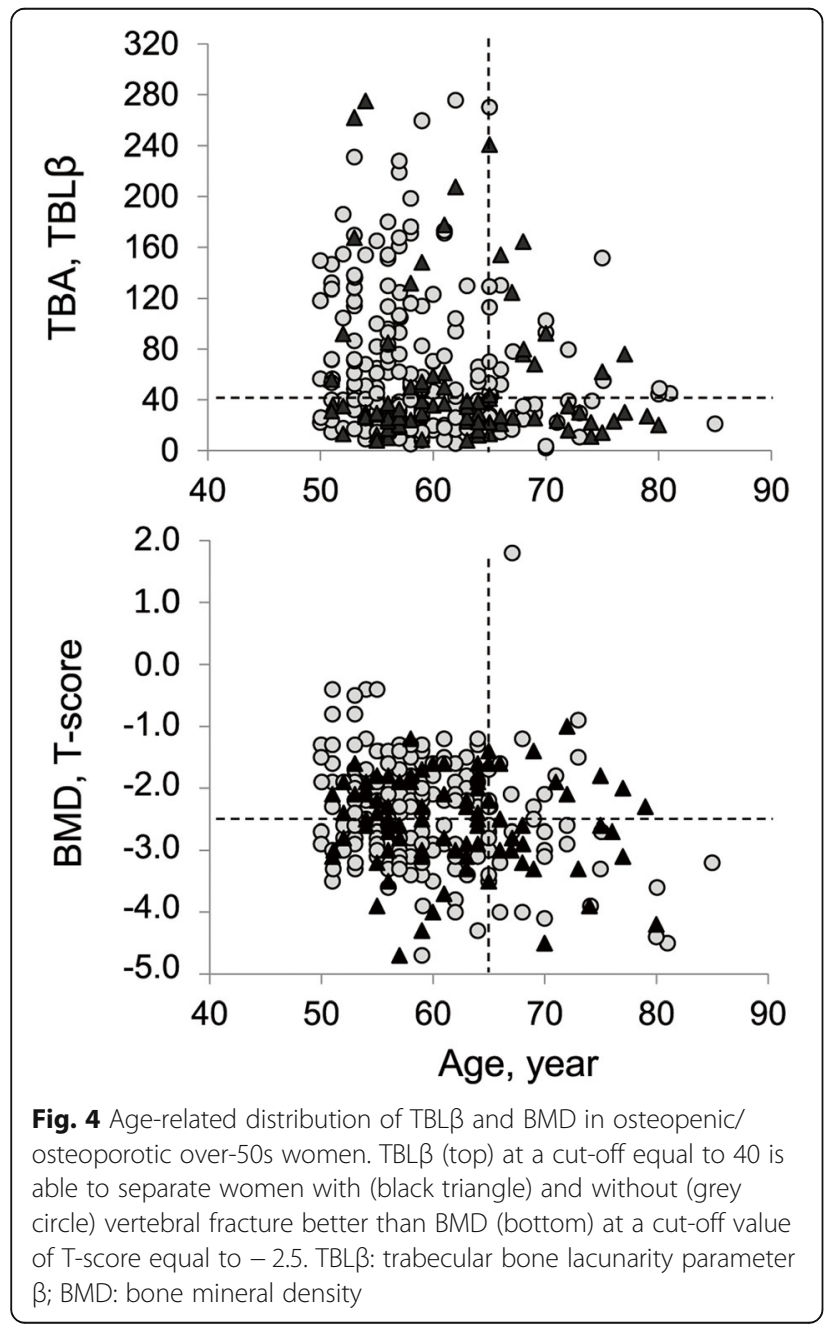

lower $(p=0.002)$ in VF+ when compared with VF- independently on BMD. In fact, osteoporotic and osteopenic patients did not show any statistical difference $(p=$ $0.795)$. A statistically significant difference $(p=0.001)$ exists between VF+ and VF- within the osteoporotic group but not within osteopenic one despite a lower value in $\mathrm{VF}+$ osteopenic patients when compared with VF- ones.

\section{Predicting bone fragility vertebral fractures by TBA and BMD}

Comparison of ROC curves for TBL $\beta$ and BMD T-score is illustrated in Fig. 5. Here we report results related to TBL $\beta$ cut-off value 40 (mean value between Youden index: 39 and median value from the whole sample: 41 ). The same cut-off value was previously observed in a pilot study. By ROC curve analysis, AUC for TBL $\beta$ was $0.63(\mathrm{z}=3.795 ; p=0.005)$, statistically higher $(p=0.032)$ than BMD T-score (threshold $=-2.5 \mathrm{SD}$ ) with an AUC equal to $0.53(\mathrm{z}=2.400 ; p=0.016)$. It is worth noting that by using BMD expressed as $\mathrm{g} / \mathrm{cm}^{2}$ we obtained comparable results (data not shown). Sensitivity, specificity, positive predictive value (precision), negative predictive value and accuracy (effectiveness) were estimated for $\mathrm{TBL} \beta$ vs. $\mathrm{BMD}$ respectively as follow: $\mathrm{SN}=0.66$ vs. $0.52 ; \mathrm{SP}=0.59$ vs. $0.54 ; \mathrm{PPV}=0.43$ vs. $0.35 ; \mathrm{NPV}=0.79$ vs. $0.71, \mathrm{ACC}=0.64$ vs. 0.54 .

OR calculated in the whole sample according to Altman [28] was equal to 2.80 for TBL $\beta$ (95\% CI: 1.6359 to 4.7433; $p<0.001$ ) corresponding to a statistically significant moderate association of TBL $\beta$ with prevalent VF while BMD T-score showed a weak association $(\mathrm{OR}=$ 1.31; $95 \%$ CI: 0.7893 to $2.1717 ; p=0.297$ ). Venn diagram (Fig. 6) of osteoporotic fracture risk factors for prevalent VF in over-50s women showed that the contribution of TBA (TBL $\beta \leq 40$, n. 58 out of $88,66 \%$ ) alone is higher than age (years $\geq 65$, n. 29, 33\%) and BMD (T-score $\leq-$ 
Table 2 TBL $\beta$ vs. BMD in over-50s women with/without bone fragility vertebral fracture

\begin{tabular}{|c|c|c|c|c|c|c|}
\hline \multirow[t]{2}{*}{ Subjects } & \multicolumn{2}{|l|}{ TBL $\beta$} & \multirow[t]{2}{*}{$P$} & \multicolumn{2}{|l|}{ BMD } & \multirow[t]{2}{*}{$P$} \\
\hline & VF- & VF+ & & VF- & VF+ & \\
\hline Overall & $51(28-101)$ & $31(23-60)$ & 0.002 & $0.895 \pm 0.107$ & $0.875 \pm 0.094$ & 0.131 \\
\hline Osteopenic & $47(23-104)$ & 38 (24-89) & 0.123 & $0.953 \pm 0.040$ & $0.972 \pm 0.071$ & 0.071 \\
\hline Osteoporotic & 52 (32-93) & $30(23-51)$ & 0.001 & $0.803 \pm 0.057$ & $0.804 \pm 0.069$ & 0.351 \\
\hline Untreated & $52(29-107)$ & $30(23-53)$ & 0.027 & $0.920 \pm 0.106$ & $0.885 \pm 0.096$ & 0.039 \\
\hline Treated & $50(26-93)$ & $31(23-62)$ & 0.078 & $0.853 \pm 0.095$ & $1.025 \pm 1.148$ & 0.280 \\
\hline
\end{tabular}

Values are median (interquartile range) for TBL $\beta$ and mean \pm standard deviation for BMD

TBL $\beta$ : trabecular bone lacunarity parameter $\beta$; BMD: bone mineral density expressed in $\mathrm{mg} / \mathrm{cm}^{2}$; VF+: with vertebral fracture; VF-: without vertebral fracture; P: Statistical significance for $p \leq 0.05$

2.5 , n. $46,52 \%)$. Combining the three risk factors would contribute to identify $86 \%$ patients at high risk of osteoporotic VF. Other risk factors responsible for prevalent VF account for $14 \%$.

We observed that VF+ subjects with TBL $\beta>40$ included several $\mathrm{T}+$ patients. Therefore, to exclude the influence of any medication on both TBA and BMD, we considered only $\mathrm{T}$ - subjects. We found that, among 156 T- patients, $69 \%$ (24 out of 35 ) were associated with a TBL $\beta \leq 40$ vs. $49 \%$ patients (17 out of 35 ) with a BMD T-score $\leq-2.5$ (Table 3). OR was 3.10 (95\% CI: 1.3919 to 6.8962; $p=0.006$ ) for TBL $\beta$ and 1.49 (95\% CI: 0.6975 to $3.1701 ; p=0.304$ ) for BMD T-score, that account for a statistically significant moderate association of TBL $\beta$ with prevalent VF against a weak not significant association for BMD T-score.

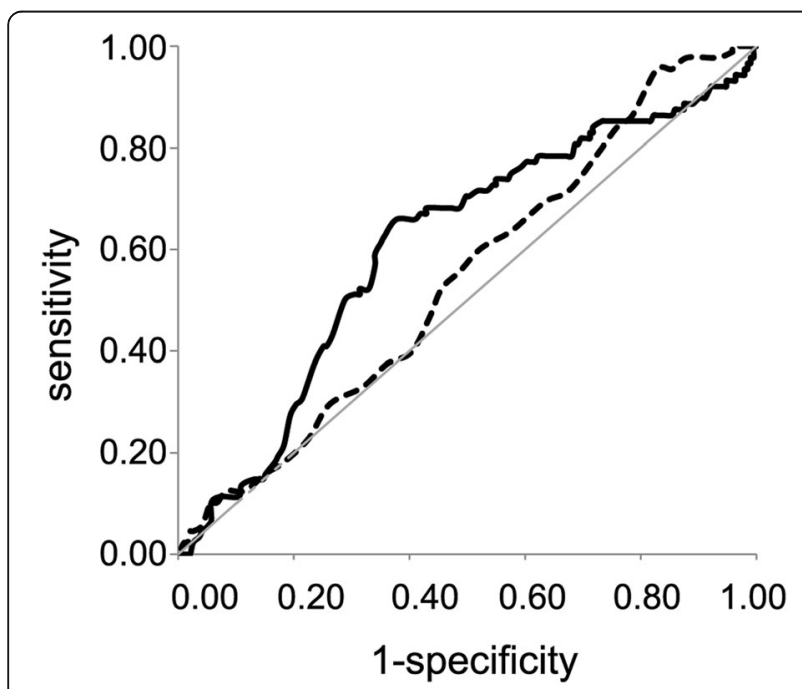

Fig. 5 Empirical ROC curves for both TBL $\beta$ (solid line) and BMD Tscore (dotted line) data sets. Light grey line represents the reference line. Each point in the curves represents true positive (sensitivity, ordinate axis) vs. false positive (1-specificity, abscissa axis) for different thresholds. ROC: receiver operating characteristics; TBL $\beta$ : trabecular bone lacunarity parameter $\beta$; BMD: bone mineral density

\section{Preliminary 1-year prospective results}

We observed that several VF- patients had a TBL $\beta \leq 40$, that is, at risk for bone fragility fracture. Preliminary results on incident VF (Table 3) showed that, among 155 patients at 1 -year follow up, $73 \%$ incident $\mathrm{VF}+$ subjects (24 out of 33) were associated with a TBL $\beta \leq 40$ at baseline vs. $55 \%$ (18 out of 33 ) for BMD T-score $\leq-2.5$. The relative risk (RR) calculated according to Altman [28] showed a statistically significant moderate association of TBL $\beta$ with incident VF ( $R R=3.00,95 \% \mathrm{CI}: 1.4902$ to 6.0210; $p=0.002)$ against a low association for BMD Tscore $(\mathrm{RR}=1.31 ; 95 \% \mathrm{CI}: 0.7148$ to $2.4136 ; p=0.380)$. It is worth noting that in $80 \mathrm{~T}$ - patients at baseline, $78 \%$ incident VF+ subjects (14 out of 18) were associated to a baseline TBL $\beta$ value $\leq 40$ vs. $50 \%$ for BMD ( 9 out of 18 ). The related $\mathrm{RR}=3.17$ (95\% CI: 1.1409 to $8.7897 ; p=$

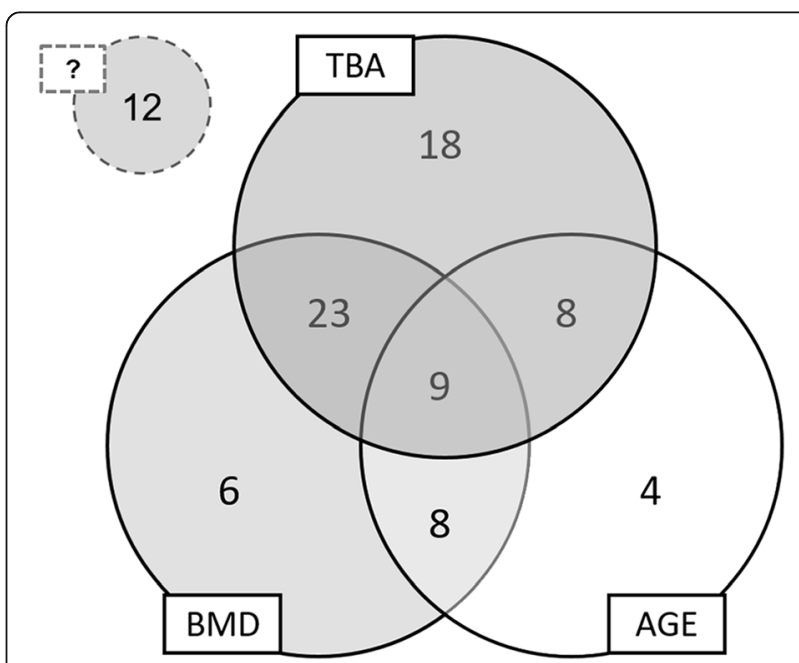

Fig. 6 Venn diagram of osteoporotic fracture risk factors for prevalent vertebral fractures in over-50s women. Contribution of TBA $(T B L \beta \leq 40,66 \%)$ alone is higher than AGE (years $\geq 65,33 \%$ ) and BMD (T-score $\leq-2.5,52 \%)$. Combining the three risk factors would contribute to identify $86 \%$ patients at high risk of osteoporotic fractures. Other risk factors (?) are responsible for prevalent fractures in 14\% women. TBA: trabecular bone microarchitecture; TBL $\beta$ : trabecular bone lacunarity parameter $\beta$; BMD: bone mineral density 
Table 3 Contribution of TBA and BMD to prevalent and incident bone fragility vertebral fractures

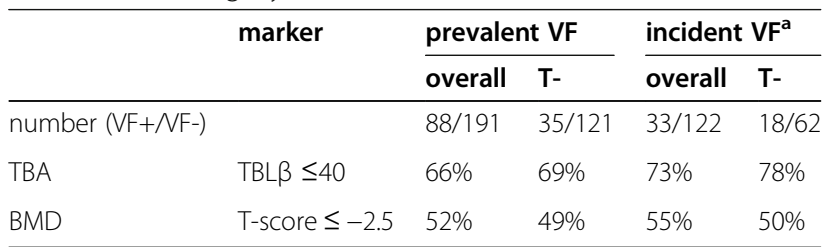

TBL $\beta$ : trabecular bone microarchitecture parameter $\beta$; BMD: bone mineral density; VF: vertebral fracture; VF+: with VF; VF-: without VF; T-: untreated at baseline

${ }^{a}$ Preliminary results from 1-year follow up

0.027 ) indicates a moderate association with incident VF against a weak association for BMD $\mathrm{T}$-score $(\mathrm{RR}=1.29$; 95\% CI: 0.5711 to $2.8946 ; p=0.544)$. Results from AR calculation in the whole sample suggest that by monitoring TBA degeneration as a risk factor for bone fragility fracture would reduce VF incidence of about $25 \%$ (AR = 0.24 ) against only $6 \%$ in the case of BMD $(A R=0.06)$.

\section{Discussion}

In this study, we present a new potential diagnostic tool useful in the management of fracture risk in osteoporosis pathology. An observational, cross-sectional, and prospective study (LOTO) on osteopenic/osteoporotic over-50s women, at risk for bone fragility fracture, was designed for diagnostic assessment of the method. It is based on fractal lacunarity texture analysis of TBA in lumbar vertebra images acquired by $1.5 \mathrm{~T}$ MRI system $[14,17,25]$. The method provides only one parameter, TBL $\beta$, particularly sensitive to TBA degeneration [14]. Baseline results from LOTO study show that TBL $\beta$ is statistically lower in subjects with prevalent VF than in non-fractured ones. In addition, TBL $\beta$ is able to discriminate between subjects with and without VF better than BMD thus becoming an index candidate of osteoporotic fracture risk assessment.

Currently, DXA is commonly used to measure BMD and predict fracture risk. However, reliable BMD prediction of osteoporotic fractures is obfuscated by the significant overlap between subjects with and without bone fragility fractures [29]. In this context, criteria recommended by WHO for osteoporosis diagnosis and related therapeutic treatments to care for the disease and prevent bone fracture deserve some discussion $[3,30,31]$ mainly as far as age and BMD as the risk factors are concerned. In Italy, as in many other industrialized countries, BMD measurement has been for long time mandatory only after 65 years of age in the absence of other risk factors [32]; nevertheless, most menopausal women do not perceive the severity of this pathology until a fracture event occurs due to the silent onset and progression of osteoporosis.
Results from LOTO study on the prevalence of bone fragility VF in relation to the two main risk factors (age $\geq$ 65 and BMD T-score $\leq-2.5$ as indicated by WHO), for primitive osteoporosis [3, 30-32] and consequent bone fragility fractures, confirm literature evidence $[3,5,29$, 31]. In particular, a relevant percentage of VF has been found in women younger than 65 years. It is worth noting that a high probability exists for women with menopausal osteoporotic VF to experience a senile osteoporotic femur fracture. On the other hand, lowering age limit is not sufficient to improve the management of primitive postmenopausal osteoporosis. In fact, BMD alone is not a good predictor of fracture risk [10] as confirmed in this study: $29 \%$ of osteopenic women have already experienced a vertebral bone fragility fracture that account for more than $50 \%$ prevalent fractures in LOTO patients.

Screening of the population at risk for bone fragility and treatment assessment of patients to prevent consequent fractures would be useful tools to increase quality of life in the elderly and to lighten the related healthcare-socio-economic impact. Noninvasive tools are necessary to characterise bone quality beyond bone mass to accurately assess the individual risk of bone fragility fracture and to evaluate the progression of osteoporosis and the efficacy of its treatment [10, 32, 33]. WHO recommended osteoporosis diagnosis be made a DXA-BMD measurement when noninvasive technologies to assess bone structure in vivo were not available yet. It is worth noting that guidelines for osteoporosis management have been already updated by introducing bone quality evaluation [32, 34, 35]. Nevertheless, BMD and age remain the main risk factors to be considered for bone fragility fracture risk. The limits of these two fracture risks can be overcome by adopting algorithms, i.e. FRAX, to better predict fracture risk and decide for osteoporosis treatment [36, 37]. FRAX, a fracture risk assessment tool, estimates the 10-year probability of hip and major osteoporotic fractures based on the individual's risk factor profile [36]. However, it has been emerging that FRAX does not add improvement in fracture risk assessment when compared to BMD in peri- and early postmenopausal women [38, 39]. DXA devices of last generation have been starting to be equipped with dedicated software for bone quality investigation. Nevertheless, this kind of technology, despite the name (TBS: trabecular bone score), cannot investigate TBA [40, 41].

The introduction of nonionizing/noninvasive tools to quantify TBA in clinical practice would complete the diagnosis of osteoporosis as defined by WHO [1]. MRIbased diagnosis could complement standard BMD methods for assessing osteoporosis and monitoring longitudinal changes. MRI, in fact, has been emerging as a useful tool in the study of osteoporosis. Several aspects 
of this technology candidate MRI as a noninvasive/nonionizing tool for in vivo study of bone tissue applicable to humans: MRI does not use ionizing radiation, allows direct acquisition of multiplanar images, and can explore bone physiology features otherwise not investigable by other imaging techniques [13]. As a matter of fact, quantitative MRI techniques, from diffusion tensor imaging (i.e. mean diffusivity and fractional anisotropy) to chemical shift imaging (i.e. T2* and quantitative susceptibility mapping), have been used to discriminate benign and malignant compressed vertebras [42, 43]. Among recent MRI methods to analyse bone tissue in osteoporosis [44, 45], most efforts have been devoting to fat fraction quantification at the spine by analysing bone marrow composition (for review see [46]). However, there are not studies on large cohorts to accomplish the transition of these methods into clinical practice, partially because of limited evidence of their usefulness in predicting fracture risk.

As far as TBA characterisation is concerned, comparing CT (computer tomography) with MR imaging, CT has the advantage of visualizing bone with higher spatial resolution, but the disadvantage of applying considerable radiation dose not applicable to the central skeleton. Bone structure measurements by MRI have been found to be similar to histological or micro-CT ones and highly correlated to HR-pQCT (high resolution-peripheral quantitative CT) [47]. TBA parameters as measured by 3.0 $\mathrm{T}$ have been found statistically better than $1.5 \mathrm{~T}$ MRI when compared with micro-CT as the reference standard. At $3.0 \mathrm{~T}$ the effect of magnetic susceptibility, responsible for apparent increase in trabecular thickness [13], is higher than that at $1.5 \mathrm{~T}[48,49]$. The apparent increase in trabecular thickness, an artefact due to magnetic susceptibility differences between bone and bone marrow, has the advantage to visualize the smallest trabeculas undetectable in normal conditions because of partial volume effect [33]. At present, most researchers use MR 3D or 2D images acquired by ether gradientecho or spin-echo techniques. This last modality has the advantage to reduce the above-mentioned magnetic susceptibility related artefact [13].

In early studies, MRI parameters of TBA were found to separate patients with and without osteoporotic fractures better than BMD [50]. Traditional morphometric parameters, such as bone volume fraction (BV/TV), trabecular bone number (Tb.N), and thickness (Tb.Th), showed superior results compared to BMD in separating fractured and non-fractured groups [51]. At present, prospective trials on osteoporotic fractures or large-scale therapeutic trials based on MRI-TBA characterisation are rare [52] while most recent studies deal with BMD alone as the main endpoint [53, 54]. However, this kind of studies would prompt up the definition of a diagnostic set of markers able to complement and/or improve fracture risk assessment based on DXA-BMD. The main obstacles to reach this goal are: limited dissemination of technology in healthcare centres, minimum protocols' standardization for both image acquisition and image processing, and the high number of parameters to characterise TBA. As a matter of fact, one most recent longitudinal study on alendronate treatment used 3.0 T MRI [55]. Image acquisition was related to mirror sites such as distal tibia, distal radius, and proximal femur. BV/TV, Tb. N, Tb. Th, and Tb. Sp (spacing) were among TBA parameters analysed. Seven additional parameters by geodesic topological analysis (GTA) were also included. Apparent Tb. N and four GTA parameters showed statistical treatment effects only in the distal tibia after 24 months when compared to BMD.

New MR imaging modalities, recently proposed for TBA characterization in both early diagnosis and treatment assessment of osteoporosis, have the limitation of using ever increasing powerful instrumentations (3, 7, and even $11 \mathrm{~T}$ ) for $2 \mathrm{D}$ or 3D TBA imaging in peripheral sites and characterisation by classic histomorphometric analyses [14]. This is mainly due to the choice of microCT as the reference standard $[47,54]$ that alienates the application in clinical setting of a promising noninvasive/nonionizing diagnostic tool.

The original and innovative proposed method, based on fractal lacunarity analysis of vertebral TBA, [14, $16,17,21,25]$ appears particularly promising. It uses 1.5 T MRI widely available in most healthcare centres and provides only one parameter particularly sensitive to TBA changes thus representing a suitable tool for an easy and fast applicability into both research and clinical practice. MRI systems with $1.5 \mathrm{~T}$ magnetic field power provide high resolution images with pixel size of about $400 \mu \mathrm{m}$. As already discussed elsewhere [14], while $\mathrm{Tb}$. Th is smaller than such a resolution $(100-300 \mu \mathrm{m})$, bone marrow spaces are larger (averagely $800-2000 \mu \mathrm{m}$ ) thus making still reasonable TBA characterisation by MRI [13, 47]. In addition, image processing and image analysis techniques allow overcoming the limits of image quality and resolution. The computational approach adopted in our method to quantify TBA deterioration by TBL $\beta$, based on fractal lacunarity texture analysis in greyscale images, overcomes the limits of image binarization process and provides one parametric result representative of an holistic characterisation of TBA, comprehensive of BV/TV, Tb. Sp, Tb. N, Tb.Th.

Baseline results from LOTO study confirm the goodness of TBL $\beta$ as an index of osteoporotic fracture risk more suitable than BMD in separate patients with and without bone fragility fractures. Introducing TBA characterisation by TBL $\beta$ into clinical practice to 
complement DXA-BMD based diagnosis would contribute to identify up to $86 \%$ women at high risk of bone fragility fracture. Preliminary results from 1-year follow up seem to be even more promising. The projection of $\mathrm{AR}$, that is the risk due to the single risk factor considered, suggests that by monitoring TBA degeneration as a risk factor for bone fragility fracture would reduce the incidence of osteoporotic fractures of $25 \%$ vs. only $6 \%$ for BMD.

It is worth noting that differences in results between whole sample and untreated group forewarn the potential of TBL $\beta$ as an index useful in the assessment of therapy efficacy. Preliminary results previously presented [56] further support the potential role TBL $\beta$ can play in monitoring the efficacy of osteoporosis drug treatment in preventing or reducing bone fragility fracture risk. This aspect needs a deeper analysis of baseline data as they are from an observational study with heterogeneity in both therapy type and time. We expect more consistent results about this aspect from LOTO study prospective phases as information on both type and time of osteoporosis medications prescribed to baseline untreated patients would be well documented. Nevertheless, dedicated pharmacologic studies are also necessary.

This study represents a first step toward clinical validation of the proposed method. Multicentric studies are desirable and phantom based setup of different MRI instrumentations from several health centres will need. It would allow establishing a common protocol of image acquisition to guarantee comparison of results by avoiding discrepancies among different manufacturers devices.

In this context, the proposed method, based on fractal lacunarity texture analysis of MRI-TBA, is easy and fast to apply thus making simple to face this kind of studies.

Improvements of the method are in progress to overcome the limit of rectangular ROI, used in this study, by adopting a circle-like shape. A circular ROI to fit the whole trabecular bone area within the inner perimeter of vertebral body would allow for a more accurate quantification of TBA deterioration.

\section{Conclusion}

TBL $\beta$ method, based on fractal lacunarity of TBA in vertebral MRI, represents a useful noninvasive/nonionizing tool to assess TBA deterioration and predict bone fragility fracture. TBL $\beta$ has also potential as an index to monitor osteoporosis therapy response. TBL $\beta$ method uses 1.5 T-MRI, widely available in clinical setting, and provides only one parameter, TBL $\beta$, to characterise TBA, thus representing an easy and fast tool promptly applicable to both clinical and research studies on osteoporosis.

\section{Abbreviations}

ACC: Accuracy; AR: Attributable risk; AUC: Area under the curve; BMD: Bone mineral density; BV/TV: Bone volume fraction; CT: Computer tomography; DXA: Dual-energy x-ray absorptiometry; FRAX: Fracture risk assessment tool; GBA: Gliding box algorithm; GTA: Geodesic topological analysis; HRPQCT: High resolution-peripheral quantitative CT; LOTO: Lacunarity Of Trabecular bone in Osteoporosis; MRI: Magnetic resonance imaging; MRX: Morphometry on conventional x-ray images; MXA: Morphometry on densitometric images; NPV: Negative predictive value; OR: Odds ratio; PPV: Positive predictive value; ROC: Receiver Operating Characteristics; RR: Relative risk; SN: Sensitivity; SP: Specificity; T+: treated subjects; T: Untreated subjects; TBA: Trabecular bone microarchitecture;

TBL $\beta$ : Trabecular bone lacunarity parameter $\beta$; Tb.N: Trabecular bone number; Tb.Sp: Trabecular bone spacing; Tb.Th: Trabecular bone thickness; VF: Vertebral fracture; VF+: with prevalent vertebral fractures.; VF-: Without vertebral fractures; WHO: World Health Organization

\section{Acknowledgements}

The LOTO study group wishes to thank Simona Giovagnetti, David Gubbini and all radiology personnel for their precious contribution to project implementation.

\section{Authors' contributions}

All authors contributed extensively to the work presented in this paper. AZ designed the study, performed experiments, and drafted the manuscript; RR and MS provided technical support and supervision on diagnostic imaging; RG and PS collected clinical data; PM provided support to image analysis and statistical analyses. All authors critically revised and approved the final version of the submitted manuscript.

\section{Funding}

This study was supported by non-competing fund 'Ricerca Corrente' from Italian Ministry of Health to IRCCS INRCA. The funder of the study had no role in study design, data collection, data analysis, data interpretation, or writing the manuscript.

\section{Availability of data and materials}

The datasets generated and analyzed during the current study are available from the corresponding author upon reasonable request.

\section{Ethics approval and consent to participate}

The LOTO study was approved by the institutional ethics committee, Comitato Etico INRCA, (committee's reference number: FiORdiLOTO SC/11/ 281) and was performed in accordance with the Declaration of Helsinki (1964) and its later amendments.

Written informed consent was received from participants prior to inclusion in the study.

\section{Consent for publication}

Not applicable.

\section{Competing interests}

The authors declare that they have no competing interests.

\section{Author details}

${ }^{1}$ Centre of Innovative Models for Ageing Care and Technology, Scientific Direction, IRCCS INRCA, Via S. Margherita 5, I-60121 Ancona, Italy. ${ }^{2}$ Medical Imaging Division, Geriatric Hospital, IRCCS INRCA, 60124 Ancona, Italy. ${ }^{3}$ Analysis Laboratory, Geriatric Hospital, IRCCS INRCA, 60124 Ancona, Italy. ${ }^{4}$ School of Science and Technology, University of Camerino, 62032 Camerino, MC, Italy. ${ }^{5}$ Rheumatology Division, Geriatric Hospital, IRCCS INRCA, 63900 Fermo, Italy.

Received: 3 July 2020 Accepted: 11 January 2021

Published online: 23 January 2021

\section{References}

1. Anonymus. Consensus development conference. Diagnosis, prophylaxis and treatment of osteoporosis. Am J Med. 1993;94:646-50. 
2. Guglielmi G, Muscarella S, Bazzocchi A. Integrated imaging approach to osteoporosis: state-of-the-art review and update. Radiographics. 2011;31: 1343-64.

3. Jordan KM, Cooper C. Epidemiology of osteoporosis. Best Pract Res Clin Rheumatol. 2002;16:795-806.

4. Johnell O, Kanis J. Epidemiology of osteoporotic fractures. Osteoporos Int. 2005;16(Suppl 2):S3-7.

5. O'Neill TW, Felsenberg D, Varlow J, Cooper C, Kanis JA, Silman AJ. The prevalence of vertebral deformity in European men and women: the European vertebral osteoporosis study. J Bone Miner Res. 1996;11: 1010-8

6. Ismail AA, O'Neill TW, Cooper C, Finn JD, Bhalla AK, Cannata JB, Delmas P, Falch JA, Felsch B, Hoszowski K, Johnell O, Diaz-Lopez JB, Lopez Vaz A, Marchand F, Raspe H, Reid DM, Todd C, Weber K, Woolf A, Reeve J, Silman AJ. Mortality associated with vertebral deformity in men and women: results from the European prospective osteoporosis study (EPOS). Osteoporos Int. 1998;8:291-7.

7. Kanis JA, Borgstrom F, De Laet C, Johansson H, Johnell O, Jonsson B, Oden A, Zethraeus N, Pfleger B, Khaltaev N. Assessment of fracture risk. Osteoporos Int. 2005;16:581-9.

8. Lindsay R, Silverman SL, Cooper C, Hanley DA, Barton I, Broy SB, Licata A Benhamou L, Geusens P, Flowers K, Stracke H, Seeman E. Risk of new vertebral fracture in the year following a fracture. JAMA. 2001;285:320-3.

9. Helgason B, Perilli E, Schileo E, Taddei F, Brynjolfsson S, Viceconti M. Mathematical relationships between bone density and mechanical properties: a literature review. Clin Biomech. 2008;23:135-46.

10. Seeman E. Is a change in bone mineral density a sensitive and specific surrogate of anti-fracture efficacy? Bone. 2007;41:308-17.

11. Dalle Carbonare L, Giannini S. Bone microarchitecture as an important determinant of bone strength. J Endocrinol Investig. 2004:27:99-105.

12. Samelson EJ, Broe KE, Xu H, Yang L, Boyd S, et al. Cortical and trabecular bone microarchitecture as an independent predictor of incident fracture risk in older women and men in the bone microarchitecture international consortium (BoMIC): a prospective study. Lancet Diabetes Endocrinol. 2019; 7:34-43.

13. Krug R, Burghardt AJ, Majumdar S, Link TM. High-resolution imaging techniques for the assessment of osteoporosis. Radiol Clin N Am. 2010;48: 601-21.

14. Zaia A. Fractal lacunarity of trabecular bone and magnetic resonance imaging: new perspectives for osteoporotic fracture risk assessment. World J Orthop. 2015;6:221-35.

15. Majumdar S. A review of magnetic resonance (MR) imaging of trabecular bone micro-architecture: contribution to the prediction of biomechanical properties and fracture prevalence. Technol Health Care. 1998;6:321-7.

16. Zaia A, Eleonori R, Maponi P, Rossi R, Murri R. Medical imaging and osteoporosis: Fractal's Lacunarity analysis of trabecular bone in MR images. In: Tsymbal A, Cunningham P, editors. Proceedings of the eighteenth IEEE symposium on computer-based medical systems. Los Alamitos CA: IEEE Computer Society Press; 2005. p. 3-8.

17. Zaia A, Eleonori R, Maponi P, Rossi R, Murri R. MR imaging and osteoporosis: fractal Lacunarity analysis of trabecular bone. IEEE Trans Inf Technol Biomed. 2006;10:484-9.

18. Mandelbrot BB. The fractal geometry of nature. New York: WH Freeman; 1982.

19. Cross SS. Fractals in pathology. J Pathol. 1997;182:1-8.

20. Lipsitz LA, Goldberger AL. Loss of 'complexity' and aging: potential applications of fractals and chaos theory to senescence. JAMA. 1992;267: 1806-9.

21. Zaia A. Osteoporosis and fracture risk: new perspectives for early diagnosis and treatment assessment. In: Mattingly BE, Pillare AC, editors. Osteoporosis: etiology, diagnosis and treatment. Hauppauge NY: Nova Science Publishers; 2009. p. 267-90.

22. Zaia A, Maponi P, Di Stefano G, Casoli T. Biocomplexity and Fractality in the search of biomarkers of aging and pathology: focus on mitochondrial DNA and Alzheimer's disease. Aging Dis. 2017;1:44-56

23. Zaia A, Maponi P, Zannotti M, Casoli T. Biocomplexity and Fractality in the search of biomarkers of aging and pathology: mitochondrial DNA profiling of Parkinson's disease. Int J Mol Sci. 2020;21:1758.

24. Dougherty G, Henebry GM. Lacunarity analysis of spatial pattern in CT images of vertebral trabecular bone for assessing osteoporosis. Med Eng Phys. 2002;24:129-38.
25. Zaia A, Rossi R, Egidi N, Maponi P. Fractal's lacunarity analysis of trabecular bone in MR images. In: Tavares J, Jorge N, editors. Computational vision and medical image processing. United States: CRC Press; 2010. p. 421-6.

26. Genant HK, Jergas M, Palermo L, Nevitt M, San Valentin R, Black D, Cummings SR. Comparison of semiquantitative visual and quantitative morphometric assessment of prevalent and incident vertebral fractures in osteoporosis. J Bone Miner Res. 1996;11:984-96.

27. Allain C, Cloitre M. Characterizing the lacunarity of random and deterministic fractal sets. Phys Rev A. 1991;44:3552-8.

28. Altman DG. Practical statistics for medical research. London: Chapman and Hall; 1991.

29. Schousboe JT. Epidemiology of vertebral fractures. J Clin Densitom. 2016;19: $8-22$

30. Olson AF. Osteoporosis. Nurse Pract. 2007;32:20-7.

31. Kanis JA, McCloskey EV, Harvey NC, Johansson H, Leslie WD. Intervention thresholds and the diagnosis of osteoporosis. J Bone Miner Res. 2015;30: 1747-53.

32. Kanis JA, McCloskey EV, Johansson H, Cooper C, Rizzoli R, Reginster JY. Scientific advisory Board of the European Society for clinical and economic aspects of osteoporosis and osteoarthritis (ESCEO), the Committee of Scientific Advisors of the international Osteoporosis Foundation (IOF). European guidance for the diagnosis and management of osteoporosis in postmenopausal women Osteoporos Int. 2013:24:23-57.

33. Silverman SL, Cummings SR, Watts NB. Consensus panel of the ASBMR, ISCD, and NOF. Recommendations for the clinical evaluation of agents for treatment of osteoporosis: consensus of an expert panel representing the American Society for Bone and Mineral Research (ASBMR), the International Society for Clinical Densitometry (ISCD), and the National Osteoporosis Foundation (NOF). J Bone Miner Res. 2008;23:159-65.

34. Lentle B, Cheung AM, Hanley DA, Leslie WD, Lyons D, Papaioannou A Atkinson S, Brown JP, Feldman S, Hodsman AB, Jamal AS, Josse RG, Kaiser SM, Kvern B, Morin S, Siminoski K. Scientific advisory Council of Osteoporosis Canada. Osteoporosis Canada 2010 guidelines for the assessment of fracture risk. Can Assoc Radiol J. 2011;62:243-50.

35. Siris ES, Adler R, Bilezikian J, Bolognese M, Dawson-Hughes B, Favus MJ, Harris ST, Jan de Beur SM, Khosla S, Lane NE, Lindsay R, Nana AD, Orwoll ES, Saag K, Silverman S, Watts NB. The clinical diagnosis of osteoporosis: a position statement from the national bone health alliance working group. Osteoporos Int. 2014;25:1439-43.

36. Kanis JA, Oden A, Johnell O, Johansson H, De Laet C, Brown J, Burckhardt P, Cooper C, Christiansen C, Cummings S, Eisman JA, Fujiwara S, Glüer C, Goltzman D, Hans D, Krieg M-A, La Croix A, McCloskey E, Mellstrom D, Melton LJ III, Pols H, Reeve J, Sanders K, Schott A-M, Silman A, Torgerson D, van Staa T, Watts NB, Yoshimura N. The use of clinical risk factors enhances the performance of BMD in the prediction of hip and osteoporotic fractures in men and women. Osteoporos Int. 2007;18:1033-46.

37. Kanis JA, Johansson H, Harvey NC, McCloskey EV. A brief history of FRAX. Arch Osteoporos. 2018;13:118.

38. Trémollieres FA, Pouillès JM, Drewniak N, Laparra J, Ribot CA, DargentMolina P. Fracture risk prediction using BMD and clinical risk factors in early postmenopausal women: sensitivity of the WHO FRAX tool. J Bone Miner Res. 2010;25:1002-9.

39. Kužma M, Hans D, Koller T, Némethová E, Jackuliak P, Killinger Z, Resch H, Payer J. Less strict intervention thresholds for the FRAX and TBS-adjusted FRAX predict clinical fractures in osteopenic postmenopausal women with no prior fractures. J Bone Miner Metab. 2018;36:580-8.

40. Bousson V, Bergot C, Sutter B, Levitz P, Cortet B. Scientific Committee of the Groupe de Recherche et d'Information Sur les Ostéoporoses. Trabecular bone score (TBS): available knowledge, clinical relevance, and future prospects. Osteoporos Int. 2012;23:1489-501.

41. Harvey NC, Glüer CC, Binkley N, McCloskey EV, Brandi M-L, Cooper C, Kendler D, Lamy O, Laslop A, Camargos BM, Reginster J-Y, Rizzoli R, Kanis JA Trabecular bone score (TBS) as a new complementary approach for osteoporosis evaluation in clinical practice. Bone. 2015;78:216-24.

42. Razek AAKA, Sherif FM. Diagnostic accuracy of diffusion tensor imaging in differentiating malignant from benign compressed vertebrae. Neuroradiology. 2019;61:1291-6.

43. Schmeel FC, Luetkens JA, Feißt A, Enkirch SJ, Endler CH, Wagenhäuser PJ, Schmeel LC, Träber F, Schild HH, Guido Matthias Kukuk GM. Quantitative evaluation of $\mathrm{T}^{*}$ relaxation times for the differentiation of acute benign and malignant vertebral body fractures. Eur J Radiol. 2018;108:59-65. 
44. Chen Y, Guo Y, Zhang X, Mei Y, Feng Y, Zhang X. Bone susceptibility mapping with MRI is an alternative and reliable biomarker of osteoporosis in postmenopausal women. Eur Radiol. 2018;28:5027-34.

45. Wu HZ, Zhang XF, Han SM, Cao L, Wen JX, Wu WJ, Gao BL. Correlation of bone mineral density with MRI T2* values in quantitative analysis of lumbar osteoporosis. Arch Osteoporos. 2020;15:18.

46. Sollmann N, Loffler MT, Kronthaler S, Bohm C, Dieckmeyer M, Ruschke S, Kirschke JS, Carballido-Gamio J, Karampinos DC, Krug R, Baum T. MRI-based quantitative osteoporosis imaging at the spine and femur. J Magn Reason Imaging. 2020. https://doi.org/10.1002/jmri.27260.

47. Krug R, Carballido-Gamio J, Burghardt AJ, Kazakia G, Hyun BH, Jobke B, Banerjee S, Huber M, Link TM, Majumdar S. Assessment of trabecular bone structure comparing magnetic resonance imaging at 3 tesla with highresolution peripheral quantitative computed tomography ex vivo and in vivo. Osteoporos Int. 2008:19:653-61.

48. Baum T, Dütsch Y, Müller D, Monetti R, Sidorenko I, Räth C, Rummeny EJ, Link TM, Bauer JS. Reproducibility of trabecular bone structure measurements of the distal radius at 1.5 and 3.0 T magnetic resonance imaging. J Comput Assist Tomogr. 2012;36:623-6.

49. Banerjee S, Han ET, Krug R, Newitt DC, Majumdar S. Application of refocused steady-state free-precession methods at 1.5 and $3 \mathrm{~T}$ to in vivo high-resolution MRI of trabecular bone: simulations and experiments. J Magn Reson Imaging. 2005;21:818-25.

50. Laib A, Newitt DC, Lu Y, Majumdar S. New model-independent measures of trabecular bone structure applied to in vivo high resolution MR images. Osteoporos Int 2002:13:130-6.

51. Griffith JF, Genant HK. Bone mass and architecture determination: state of the art. Best Pract Res Clin Endocrinol Metab. 2008;22:737-64.

52. Folkesson J, Goldenstein J, Carballido-Gamio J, Kazakia G, Burghardt AJ, Rodriguez A, Krug R, de Papp AE, Link TM, Majumdar S. Longitudinal evaluation of the effects of alendronate on MRI bone microarchitecture in postmenopausal osteopenic women. Bone. 2011;48:611-21.

53. Ebina K, Hashimoto J, Kashii M, Hirao M, Kaneshiro S, Noguchi T, Tsukamoto $Y$, Yoshikawa $H$. The effects of switching daily teriparatide to oral bisphosphonates or denosumab in patients with primary osteoporosis. $J$ Bone Miner Metab. 2017;35:91-8.

54. Tanaka S, Mori S, Hagino H, Sugimoto T. Design of a randomized trial of teriparatide followed by alendronate: Japanese osteoporosis intervention Trial-05 (JOINT-05). J Bone Miner Metab. 2020;38:412-7.

55. Cohen A, Stein EM, Recker RR, Lappe JM, Dempster DW, Zhou H, Cremers S, McMahon DJ, Nickolas TL, Müller R, Zwahlen A, Young P, Stubby J, Shane E. Teriparatide for idiopathic osteoporosis in premenopausal women: a pilot study. J Clin Endocrinol Metab. 2013;98:1971-81.

56. Zaia A, Rossi R, Galeazzi R, Scendoni P. Lacunarity of trabecular bone microarchitecture, $T B L \beta$, as a predictor of bone fragility fracture and potential index of osteoporosis treatment efficacy. The LOTO study [Abstract]. Ann Rheum Dis. 2017;76(Suppl 2):690.

\section{Publisher's Note}

Springer Nature remains neutral with regard to jurisdictional claims in published maps and institutional affiliations.

Ready to submit your research? Choose BMC and benefit from:

- fast, convenient online submission

- thorough peer review by experienced researchers in your field

- rapid publication on acceptance

- support for research data, including large and complex data types

- gold Open Access which fosters wider collaboration and increased citations

- maximum visibility for your research: over $100 \mathrm{M}$ website views per year

At $\mathrm{BMC}$, research is always in progress.

Learn more biomedcentral.com/submissions 